\title{
Large-Scale Distribution of Arrival Directions of Cosmic Rays Detected at the Pierre Auger Observatory and the Telescope Array above $10^{19} \mathrm{eV}$
}

\author{
Olivier Deligny ${ }^{* a}$ for the Pierre Auger Collaboration ${ }^{b}$ and Telescope Array \\ Collaboration ${ }^{c}$ \\ ${ }^{a}$ CNRS/IN2P3 - IPN Orsay, France \\ ${ }^{b}$ Observatorio Pierre Auger, Av. San Martín Norte 304, 5613 Malargüe, Argentina \\ E-mail: auger_spokespersons@fnal.gov \\ Full author list: http://www.auger.org/archive/authors_2015_06.html [www.auger.org] \\ ${ }^{c}$ Telescope Array Project, 201 James Fletcher Bldg, 115 S. 1400 East, Salt Lake City, UT \\ 84112-0830, USA \\ E-mail: ta-icrc@cosmic.utah.edu \\ Full author list: \\ http://www.telescopearray.org/index.php/research/publications/conference-proceedings \\ [www.telescopearray.org]
}

\begin{abstract}
The large-scale distribution of arrival directions of high-energy cosmic rays is a key observable in attempts to understanding their origin. The dipole and quadrupole moments are of special interest in revealing potential anisotropies. An unambiguous measurement of these moments as well as of the full set of spherical harmonic coefficients requires full-sky coverage. This can be achieved by combining data from observatories located in both the northern and southern hemispheres. To this end, a joint analysis using data recorded at the Pierre Auger Observatory and the Telescope Array above $10^{19} \mathrm{eV}$ has been performed. Thanks to the full-sky coverage, the measurement of the dipole moment reported in this study does not rely on any assumption on the underlying flux of cosmic rays. As well, the resolution on the quadrupole and higher order moments is the best ever obtained. The resulting multipolar expansion of the flux of cosmic rays allows a series of anisotropy searches to be performed, and in particular to report on the first angular power spectrum of cosmic rays. This allows a comprehensive description of the angular distribution of cosmic rays above $10^{19} \mathrm{eV}$.
\end{abstract}

The 34th International Cosmic Ray Conference,

30 July- 6 August, 2015

The Hague, The Netherlands

\footnotetext{
* Speaker.
} 
Introduction. Above $10^{19} \mathrm{eV}$, the flux of Ultra-High Energy Cosmic Rays (UHECRs) is expected to be of extragalactic origin. Although the actual sources of UHECRs are still to be identified, their distribution in the sky is expected to follow, to some extent, the large-scale structure of the matter in the Universe. Due to scattering in magnetic fields, the anisotropy imprinted upon the distribution of arrival directions is mainly expected at large scales even for energies as large as $10^{19} \mathrm{eV}$. A non-zero dipole moment is naturally expected for diffusive propagation of UHECRs from nearby sources, leading to a cosmic-ray density gradient embedding the observer. On the other hand, excesses along a plane, for instance the super-Galactic one, would be detectable as a prominent quadrupole. The dipole and the quadrupole moments are thus of special interest, but an access to the full set of multipoles is relevant to characterize departures from isotropy at all scales.

Recently, the full-sky multipole coefficients of the UHECR flux have been measured for the first time by the Pierre Auger and Telescope Array collaborations using a joint data set with energies above $10^{19} \mathrm{eV}$ [1]. No significant deviations from isotropic expectations were found, and upper limits on the amplitudes of the dipole and quadrupole moments were reported as a function of the direction in the sky, varying between $8 \%$ and $13 \%$ for the dipole and between $7.5 \%$ and $10 \%$ for a symmetric quadrupole. In the meanwhile, another recent report from the Pierre Auger Collaboration based on an enlarged data set has indicated an amplitude for the first harmonic in right ascension of $(4.4 \pm 1.0) \%$ with a chance probability of $6.4 \times 10^{-5}$ for events with energies larger than $8 \times 10^{18} \mathrm{eV}$ [2]. Under the assumption that the only significant contribution to the anisotropy is from the dipolar component, this observation corresponds to a dipole of amplitude $(7.3 \pm 1.5) \%$ pointing to $\left(95^{\circ} \pm 13^{\circ}\right)$ in right ascension and $\left(-39^{\circ} \pm 13^{\circ}\right)$ in declination.

The aim of this joint study is to search for anisotropy with full-sky coverage in a similar way as reported in [1], by including one additional year of data recorded at the Telescope Array and by extending the zenithal range of the data recorded at the Pierre Auger Observatory from $\left[0^{\circ}-60^{\circ}\right]$ to $\left[0^{\circ}-80^{\circ}\right]$. A detailed description of the estimation of spherical harmonic coefficients when combining data from Auger and Telescope Array is available in [1], so that only basic principles are summarized in this report. The extension of the zenithal range in the Auger data set provides a larger overlap of the field of view of the two experiments compared to the previous report. This allows a significant improvement of the resolution on the dipole moment. The joint data set considered in this study consists of events above $10^{19} \mathrm{eV}$ recorded at the Telescope Array from May 2008 to May 2014 with an exposure of $7,250 \mathrm{~km}^{2} \mathrm{yr}$ sr with zenith angles less than $55^{\circ}$, and at the Pierre Auger Observatory from 1 Jan. 2004 to 31 Dec. 2013 with an exposure of 48,029 km² $\mathrm{yr}$ sr with zenith angles less than $80^{\circ}$.

The full-sky directional exposure. The directional exposure $\omega(\mathbf{n})$ provides the effective timeintegrated collecting area for a flux from each direction of the sky. Since the energy threshold of $10^{19} \mathrm{eV}$ guarantees that both experiments are fully efficient in their respective zenith range, the directional exposure relies only on the geometrical acceptance [3]. The functions $\omega_{i}(\delta)$ of each experiment are shown in fig. 1 . Given the respective latitudes of both observatories and with the maximum zenith angle used here, full-sky coverage is naturally achieved when summing both functions. Also, it is to be noted that a common band of declination, namely $-15^{\circ} \leq \delta \leq 45^{\circ}$, is covered by both experiments.

In principle, the combined directional exposure of the two experiments should be simply the 


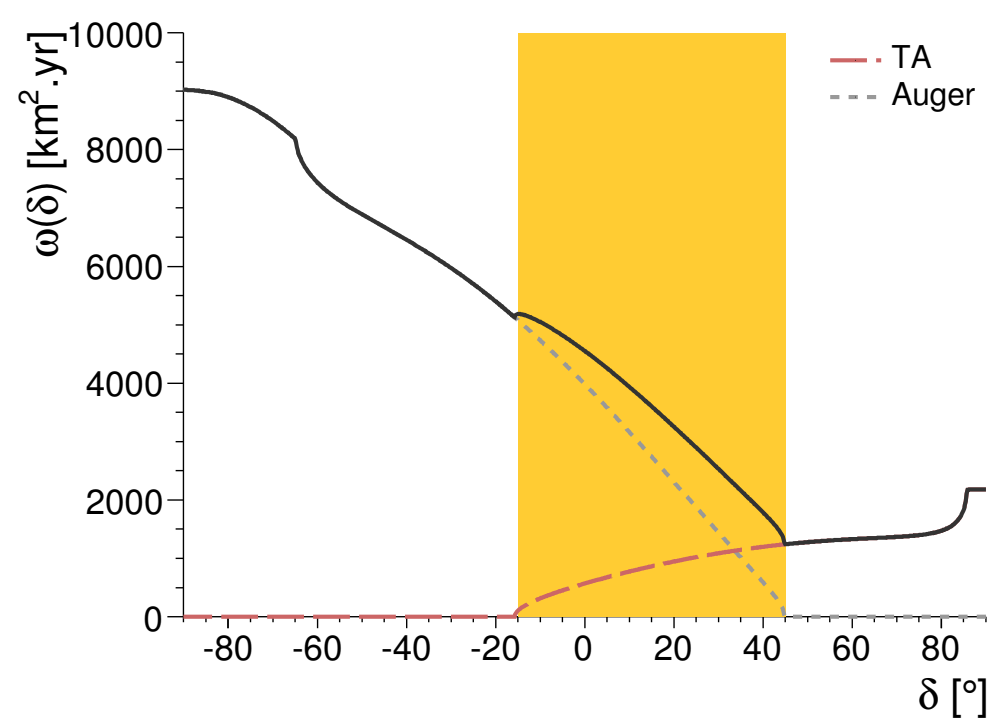

Figure 1: Directional exposure above $10^{19} \mathrm{eV}$ as obtained by summing the nominal individual ones of the Telescope Array and the Pierre Auger Observatory, as a function of the declination. The overlapping sky region is indicated by the yellow band.

sum of the individual ones. However, individual exposures have here to be re-weighted by some factor $b$ due to the unavoidable uncertainty in the relative exposures of the experiments:

$$
\omega(\mathbf{n} ; b)=\omega_{\mathrm{TA}}(\mathbf{n})+b \omega_{\text {Auger }}(\mathbf{n}) .
$$

Written in this way, $b$ is a dimensionless parameter of order unity arbitrarily chosen to re-weight the directional exposure of the Pierre Auger Observatory relative to the one of the Telescope Array. In practice, only an estimation $\bar{b}$ of the factor $b$ can be obtained, so that only an estimation of the directional exposure $\bar{\omega}(\mathbf{n}) \equiv \omega(\mathbf{n} ; \bar{b})$ can be achieved through eqn. 1 . In addition, although the techniques for assigning energies to events are nearly the same, there are differences as to how the primary energies are derived at the Pierre Auger Observatory and the Telescope Array. Currently, systematic uncertainties in the energy scale of both experiments amount to about $14 \%$ and $21 \%$ respectively $[4,5]$. Such a potential shift in energy leads to different counting rates above some fixed energy threshold, which induces fake anisotropies in a similar way to the ones resulting from a shift in the relative exposures of the experiments. The parameter $b$ can thus be viewed as an effective correction which absorbs any kind of systematic uncertainties in the relative exposures, whatever the sources of these uncertainties ${ }^{1}$.

Estimation of the spherical harmonic coefficients. The flux of cosmic rays $\Phi(\mathbf{n})$ can be decomposed in terms of a multipolar expansion onto the spherical harmonics $Y_{\ell m}(\mathbf{n})$,

$$
\Phi(\mathbf{n})=\sum_{\ell \geq 0} \sum_{m=-\ell}^{\ell} a_{\ell m} Y_{\ell m}(\mathbf{n})
$$

\footnotetext{
${ }^{1}$ Note that variations of the exposure with time due to unavoidable changes in the experimental conditions induce a dependence of the exposure functions in right ascension. These variations are however neglected in this analysis, given the much larger statistical uncertainties due to the overall small number of events above $10^{19} \mathrm{eV}$.
} 
Any anisotropy fingerprint is encoded in the multipoles $a_{\ell m}$. Non-zero amplitudes in the $\ell$ modes arise from variations of the flux on an angular scale $\simeq 1 / \ell$ radians. The observed angular distribution of cosmic rays, $\mathrm{d} N / \mathrm{d} \Omega$, can be naturally modeled as the sum of Dirac functions on the surface of the unit sphere the arguments of which are the arrival directions $\left\{\mathbf{n}_{1}, \ldots, \mathbf{n}_{N}\right\}$ of the events,

$$
\frac{\mathrm{d} N(\mathbf{n})}{\mathrm{d} \Omega}=\sum_{i=1}^{N} \delta\left(\mathbf{n}, \mathbf{n}_{i}\right)
$$

Here, arrival directions are expressed in the equatorial coordinate system (declination $\delta$ and right ascension $\alpha$ ) since this is the most natural one tied to the Earth in describing the directional exposure of any experiment. With full-sky but non-uniform coverage, the customary recipe for decoupling directional exposure effects from anisotropy ones consists in weighting the observed angular distribution by the inverse of the relative directional exposure function [3]:

$$
\frac{\mathrm{d} \tilde{N}(\mathbf{n})}{\mathrm{d} \Omega}=\frac{1}{\bar{\omega}_{r}(\mathbf{n})} \frac{\mathrm{d} N(\mathbf{n})}{\mathrm{d} \Omega} .
$$

The relative directional exposure $\bar{\omega}_{r}$ is a dimensionless function normalized to unity at its maximum. In turn, when combining the exposure of the two experiments with an unbiased estimator of $b$, the recovered coefficients defined as

$$
\bar{a}_{\ell m}=\int_{4 \pi} \mathrm{d} \Omega \frac{\mathrm{d} \tilde{N}(\mathbf{n})}{\mathrm{d} \Omega} Y_{\ell m}(\mathbf{n})=\sum_{i=1}^{N} \frac{Y_{\ell m}\left(\mathbf{n}_{i}\right)}{\bar{\omega}_{r}\left(\mathbf{n}_{i}\right)},
$$

provide unbiased estimators of the underlying $a_{\ell m}$ multipoles. Under reasonable assumptions, the resolution $\sigma_{\ell m}$ on each $a_{\ell m}$ multipole can be shown to be the sum of a first term reflecting the Poisson fluctuations induced by the finite number of events and of a second term reflecting the uncertainty in the relative exposures of the two experiments through the uncertainty to estimate the $b$ parameter. This term mainly impacts the resolution in the dipole coefficient $a_{10}$, while it has a small influence on the quadrupole coefficient $a_{20}$ and a marginal one on higher order moments $\left\{a_{\ell 0}\right\}_{\ell \geq 3}$.

The Joint-Analysis Method. The band of declinations between $-15^{\circ}$ and $45^{\circ}$ is exposed to the fields of view of both experiments. This overlapping region can be used for designing a procedure to get simultaneously a relevant estimate of the parameter $b$ and of the multipole coefficients $a_{\ell m}$ through an iteration method.

Considering as a first approximation the flux $\Phi(\mathbf{n})$ as isotropic, the overlapping region denoted by $\Delta \Omega$ can be utilized to derive a first estimate $\bar{b}^{(0)}$ of the $b$ factor by requiring the observed fluxes of both experiments to be identical in this particular region:

$$
\bar{b}^{(0)}=\frac{\Delta N_{\text {Auger }}}{\Delta N_{\mathrm{TA}}} \frac{\int_{\Delta \Omega} \mathrm{d} \Omega \omega_{\mathrm{TA}}(\mathbf{n})}{\int_{\Delta \Omega} \mathrm{d} \Omega \omega_{\text {Auger }}(\mathbf{n})},
$$

with $\Delta N_{\text {Auger }}$ and $\Delta N_{\mathrm{TA}}$ the number of events observed in the overlapping region in each experiment. Then, inserting $\bar{b}^{(0)}$ into $\bar{\omega}$, 'zero-order' $\bar{a}_{\ell m}^{(0)}$ coefficients can be obtained. This set of coefficients is only a rough estimation, due to the limiting assumption on the flux (isotropy). 

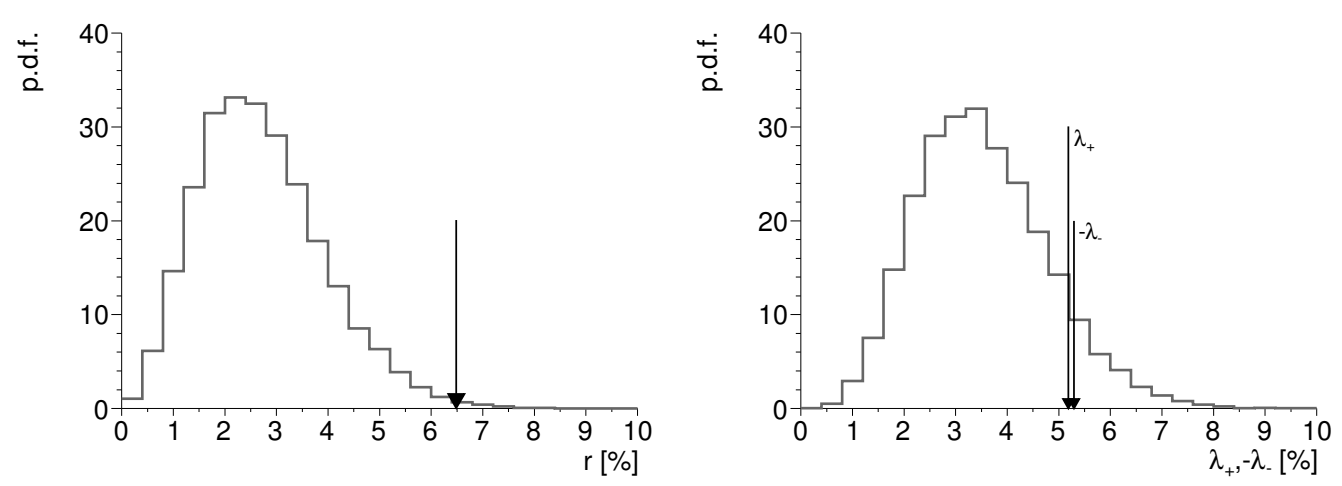

Figure 2: Measured amplitudes for the dipole vector (left) and the quadrupole tensor (right), together with the distributions expected from statistical fluctuations of isotropy.

On the other hand, the expected number of events in the common band for each observatory, $\Delta N_{\mathrm{TA}}^{\mathrm{exp}}$ and $\Delta N_{\text {Auger }}^{\mathrm{exp}}$, can be expressed from the underlying flux $\Phi(\mathbf{n})$ as

$$
\begin{aligned}
\Delta N_{\mathrm{TA}}^{\exp } & =\int_{\Delta \Omega} \mathrm{d} \Omega \Phi(\mathbf{n}) \omega_{\mathrm{TA}}(\mathbf{n}), \\
\Delta N_{\text {Auger }}^{\exp } & =\int_{\Delta \Omega} \mathrm{d} \Omega \Phi(\mathbf{n}) \omega_{\text {Auger }}(\mathbf{n}) .
\end{aligned}
$$

From eqns. 7, and from the set of $\bar{a}_{\ell m}^{(0)}$ coefficients, an iterative procedure estimating at the same time $b$ and the set of $a_{\ell m}$ coefficients can be constructed as

$$
\bar{b}^{(k+1)}=\frac{\Delta N_{\text {Auger }}}{\Delta N_{\mathrm{TA}}} \frac{\int_{\Delta \Omega} \mathrm{d} \Omega \bar{\Phi}^{(k)}(\mathbf{n}) \omega_{\mathrm{TA}}(\mathbf{n})}{\int_{\Delta \Omega} \mathrm{d} \Omega \bar{\Phi}^{(k)}(\mathbf{n}) \omega_{\text {Auger }}(\mathbf{n})},
$$

where $\bar{\Phi}^{(k)}$ is the flux estimated with the set of $\bar{a}_{\ell m}^{(k)}$ coefficients. This iterative procedure has been shown to provide unbiased estimators of the multipole coefficients without any assumptions on the flux [1].

Note that the resolution on the $b$ parameter turns out to be $2.1 \%$ in the present study, compared to $3.9 \%$ in the previous report [1]. This offers a much better resolution in estimating the $a_{10}$ coefficient. Part of this improvement is due to the statistics increased by $\simeq 46 \%$. The other factor is provided by the extended declination band exposed to the Auger and Telescope Array fields of view thanks to the use of horizontal events in the Auger data set.

Low-order multipoles. All analyses reported hereafter are based on a joint data set consisting of events with energies in excess of $10^{19} \mathrm{eV}$ in terms of the energy scale used at the Telescope Array by evaluating in the Auger data set the energy threshold which guarantees equal fluxes for both experiments. We are thus left here with 2,560 events (1,703 in the common band) above $10^{19} \mathrm{eV}$ from the Telescope Array and 16,835 (5,885 in the common band) above $8.8 \times 10^{18} \mathrm{eV}$ from the Pierre Auger Observatory. After iterations, the coefficient $b$ is $b=0.96$.

Although the full set of spherical harmonic moments is needed to characterise any departure from isotropy at any scale, the dipole and quadrupole moments are of special interest. For that 


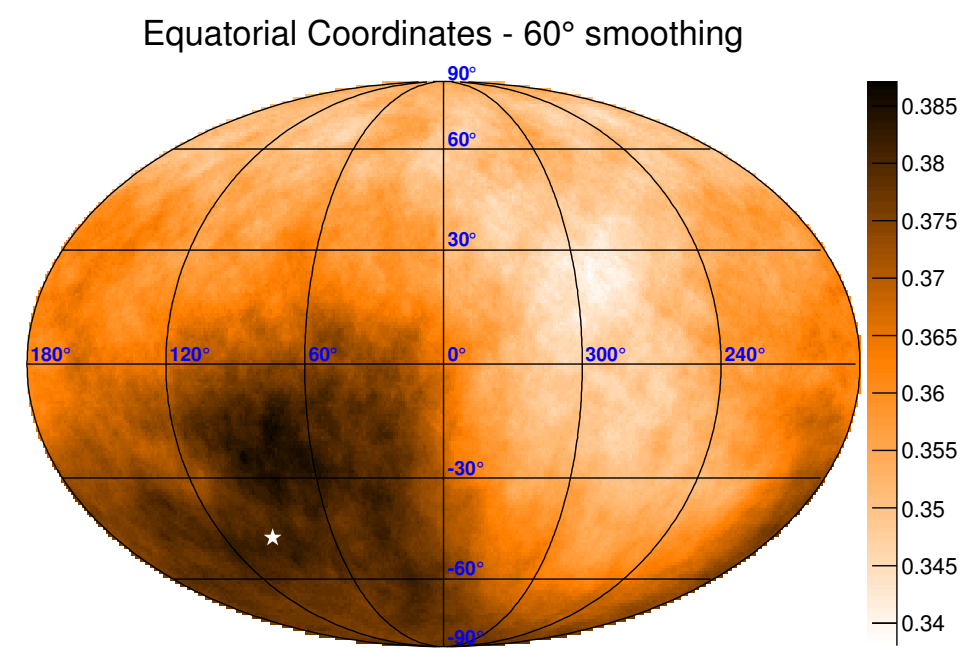

Figure 3: Sky map in equatorial coordinates of the average flux smoothed out at a $60^{\circ}$ angular scale above $10^{19} \mathrm{eV}$ in $\mathrm{km}^{-2} \mathrm{yr}^{-1} \mathrm{sr}^{-1}$ units.

reason, a special emphasis is given here to these low-order moments, in terms of a more traditional and geometric representation than the raw result of the multipole moments. The dipole moment can be fully characterized by a vector with an amplitude $r$ and the two angles $\left\{\delta_{\mathrm{d}}, \alpha_{\mathrm{d}}\right\}$ of the unit vector $\mathbf{d}$. The quadrupole, on the other hand, can be fully determined by two independent amplitudes $\left\{\lambda_{+}, \lambda_{-}\right\}$, two angles $\left\{\delta_{\mathrm{q}_{+}}, \alpha_{\mathrm{q}_{+}}\right\}$defining the orientation of a unit vector $\mathbf{q}_{+}$, and one additional angle $\alpha_{q_{-}}$defining the directions of another unit vector $\mathbf{q}_{-}$in the orthogonal plane to $\mathbf{q}_{+}$. The full description is completed by means of a third unit vector $\mathbf{q}_{0}$, orthogonal to both $\mathbf{q}_{+}$ and $\mathbf{q}_{-}$, and with a corresponding amplitude such that the traceless condition $\lambda_{+}+\lambda_{0}+\lambda_{-}=0$ is satisfied. The parameterisation of the low-order moments of the flux is then written in a convenient and intuitive way as

$$
\Phi(\mathbf{n})=\frac{\Phi_{0}}{4 \pi}\left(1+r \mathbf{d} \cdot \mathbf{n}+\lambda_{+}\left(\mathbf{q}_{+} \cdot \mathbf{n}\right)^{2}+\lambda_{0}\left(\mathbf{q}_{0} \cdot \mathbf{n}\right)^{2}+\lambda_{-}\left(\mathbf{q}_{-} \cdot \mathbf{n}\right)^{2}+\cdots\right) .
$$

The distributions of amplitudes obtained from statistical fluctuations of simulated isotropic samples are shown in fig. 2. The measured values are indicated by the superimposed arrows. The dipole amplitude is observed to be $(6.5 \pm 1.9) \%$ with a chance probability of $5 \times 10^{-3}$, pointing to $\left(93^{\circ} \pm\right.$ $24)$ in right ascension and $\left(-46^{\circ} \pm 18\right)$ in declination. Compared to the previous report in [1], the improved sensitivity in the dipole moment is primarily explained by the improved resolution on the $b$ parameter thanks to the larger common band $\Delta \Omega$, and by the increased exposure/statistics. On the other hand, the quadrupole amplitudes are observed to be within statistical fluctuations expected from isotropic samples. Overall, these results are in agreement with the ones reported in [2] without any assumption on the underlying flux of UHECRs.

To visualise the recovered dipole moment, an average flux smoothed out at an angular scale $\Theta$ per solid angle unit can be derived using the joint data set in the following way:

$$
\langle\Phi(\mathbf{n})\rangle_{\Theta}=\frac{1}{\int_{\Theta} \mathrm{d} \mathbf{n}} \int_{\Theta} \mathrm{d} \mathbf{n}^{\prime} f\left(\mathbf{n}, \mathbf{n}^{\prime}\right) \frac{1}{\bar{\omega}\left(\mathbf{n}^{\prime}\right)} \frac{\mathrm{d} N\left(\mathbf{n}^{\prime}\right)}{\mathrm{d} \Omega},
$$




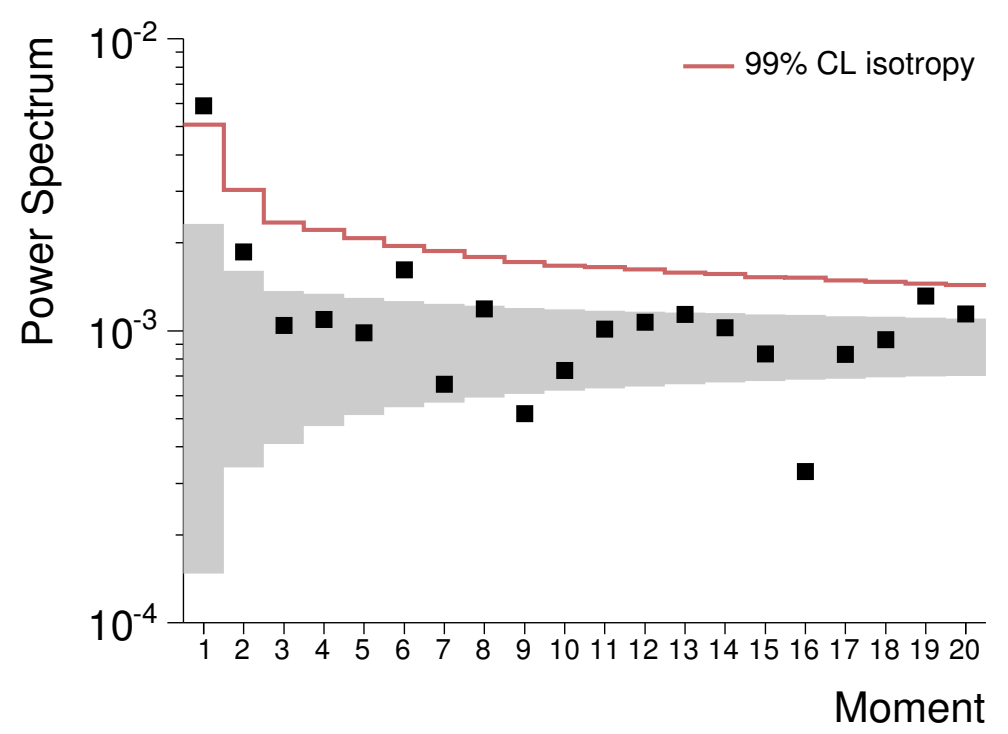

Figure 4: Angular power spectrum.

with $f$ the top-hat filter function at the angular scale $\Theta$. This average flux is displayed using the Mollweide projection in fig. 3 , in $\mathrm{km}^{-2} \mathrm{yr}^{-1} \mathrm{sr}^{-1}$ units. This map is drawn in equatorial coordinates. To exhibit the dipole structure, the angular window is chosen to be $\Theta=60^{\circ}$. The direction of the reconstructed dipole is shown as the white star.

Angular power spectrum. The angular power spectrum $C_{\ell}$ is a coordinate-independent quantity, defined as the average $\left|a_{\ell m}\right|^{2}$ as a function of $\ell$,

$$
C_{\ell}=\frac{1}{2 \ell+1} \sum_{m=-\ell}^{\ell}\left|a_{\ell m}\right|^{2}
$$

In the same way as the multipole coefficients, any significant anisotropy of the angular distribution over scales near $1 / \ell$ radians would be captured in a non-zero power in the mode $\ell$. Although the exhaustive information of the distribution of arrival directions is encoded in the full set of multipole coefficients, the characterisation of any important overall property of the anisotropy is hard to handle in a summary plot from this set of coefficients. Conversely, the angular power spectrum does provide such a summary plot. In addition, it is possible that for some fixed mode numbers $\ell$, all individual $a_{\ell m}$ coefficients do not stand above the background noise but meanwhile do so once summed quadratically.

From the set of estimated coefficients $\bar{a}_{\ell m}$, the measured power spectrum is shown in fig. 4 . The gray band stands for the RMS of power around the mean values expected from an isotropic distribution, while the solid line stands for the $99 \%$ confidence level upper bounds that would result from fluctuations of an isotropic distribution. The dipole moment is observed to stand out from the background noise, with a chance probability of $5 \times 10^{-3}$. Beyond the dipole, no other multipole deviates from expected fluctuations at $99 \% \mathrm{CL}$ in an isotropic flux. 
Outlook. In this work, an entire mapping of the celestial sphere has been achieved by combining data sets recorded at the Pierre Auger Observatory and the Telescope Array above $10^{19} \mathrm{eV}$. Throughout the series of anisotropy searches performed, a dipole moment with an amplitude $r=(6.5 \pm 1.9) \%$ is captured with a chance probability of $5 \times 10^{-3}$, while no other deviation from isotropy can be revealed at smaller angular scales.

Large-scale anisotropies of cosmic rays with energies in excess of $10^{19} \mathrm{eV}$ are closely connected to the sources and the propagation mode of extragalactic UHECRs, see e.g. [8, 9]. Due to scattering in the extragalactic magnetic fields, large deflections are expected even at such high energies for field amplitudes ranging in few nanogauss and extended over coherence lengths of the order of one megaparsec, or even for lower amplitudes if the electric charge of UHECRs is large. For sources distributed in a similar way to the matter in the Universe, the angular distribution of UHECRs is then expected to be influenced by the contribution of nearby sources, so that the Milky Way should be embedded into a density gradient of cosmic rays that should lead to at least a dipole moment. The contribution of nearby sources is even expected to become dominant as the energy of cosmic rays increases due to the reduction of the horizon of UHECR induced by energy losses more important at higher energies.

Once folded through the Galactic magnetic field, the dipole pattern expected from this mechanism is transformed into a more complex structure presumably described by a lower dipole amplitude and higher-order multipoles. However, in these scenarios, the dipole moment could remain the only one at reach within the sensitivity of the current generation of experiments. On the other hand, the detection of significant multipole moments beyond the dipole one could be suggestive of non-diffusive propagation of UHECRs from sources distributed in a non-isotropic way.

Future work will profit from the increased statistics, allowing us to uncover the angular distribution of UHECRs on the entire sky. This will provide further constraints helping to understand the origin of UHECRs.

\section{References}

[1] The Pierre Auger and Telescope Array Collaborations, ApJ 794 (2014) 172

[2] The Pierre Auger Collaboration, ApJ 802 (2015) 111

[3] P. Sommers, Astropart. Phys. 14 (2001) 271

[4] T. Abu Zayyad et al. (The Telescope Array Collaboration), Astropart. Phys. 48 (2013) 15

[5] V. Verzi for the Pierre Auger Collaboration, Proc. 33rd ICRC, Rio de Janeiro, Brazil (2013) [arXiv:1307.5059]

[6] The Pierre Auger Collaboration, JCAP 08 (2014) 019

[7] P. Billoir, O. Deligny, JCAP 02 (2008) 009

[8] D. Harari, S. Mollerach, E. Roulet, Phys. Rev. D 89 (2014) 123001

[9] P. Tinyakov, F. Urban, J. Exp. Theor. Phys. 1203 (2015) 533 